\title{
The relationship between 'normal' fluid retention in women and idiopathic oedema
}

\author{
David W. Denning,, *Matthew G. Dunnigan, ${ }^{1}$ Janet Tillman, ${ }^{2}$ Jonathan A. \\ Davis $^{3}$ and Colin A. Forrest ${ }^{3}$
}

Departments of ${ }^{1}$ Medicine, ${ }^{2}$ Biochemistry and ${ }^{3}$ Gynaecology, Stobhill General Hospital, Glasgow G21 $3 U W$, Scotland, UK.

\begin{abstract}
Summary: A sample of women attending a gynaecological outpatient clinic were examined for symptoms and objective signs of fluid retention. Patients completed a questionnaire on symptoms suggesting fluid retention and recorded daily weight and abdominal girth variation. Daily weight variation varied from 0-9 pounds (mode 2 pounds) with no discernable difference between premenstrual and intermenstrual variation. Daily girth variation varied from 0-6 inches (mode 1 inch). Symptoms of breast swelling were more common premenstrually, finger/hand and ankle swelling intermenstrually and abdominal swelling occurred with equal frequency in both periods. No correlation between symptoms and weight variation was seen although abdominal swelling and girth variation were associated $(P<0.04)$.

The results indicate that symptoms of mild fluid retention and of diurnal weight and abdominal girth variation are part of the everyday experience of our study population. There is no clear-cut separation between 'normal' and 'abnormal' fluid retention (idiopathic oedema, periodic oedema, fluid retention syndrome); the latter may be an exaggeration of normal fluid-retaining mechanisms common to most women or may represent a pathological state. An approach which evaluates individual risk factors and the severity of fluid retention in each patient is recommended.
\end{abstract}

\section{Introduction}

Idiopathic oedema (also known as cyclical oedema, periodic oedema and the fluid retention syndrome) is a poorly understood syndrome occurring almost exclusively in women. ${ }^{1-3}$ It is characterized by complaints of intermittent swelling of the face, trunk and limbs and by weight variation unrelated to the menstrual cycle. The condition is associated with obesity, diabetes mellitus and hypothyroidism $^{3}$ and many patients are psychologically abnormal. ${ }^{4}$ Diuretics are commonly prescribed with variable efficacy and a few patients become diuretic abusers. ${ }^{6}$ There is evidence of increased capillary permeability in idiopathic oedema which leads to extravasation of fluid from the vascular compartment in the upright posture with secondary retention of sodium and water through the renin-angiotensin-aldosterone pathway.

In our experience, patients may be referred to gynaecological outpatient clinics with symptoms of previously unrecognized fluid retention. Diagnosis

Correspondence and *present address: D.W. Denning M.R.C.P., D.C.H., Division of Infectious Diseases, Department of Medicine, Santa Clara Valley Medical Center, Stanford University, San Jose, California 95128, USA.

Accepted: 3 January 1990 is based on the clinical history and the exclusion of cardiac, renal, hypoproteinaemic and obstructive causes of oedema by appropriate investigations. The most characteristic clinical feature is diurnal weight gain. Thorn's statement that an increase in weight of more than $3 \mathrm{lb}(1.4 \mathrm{~kg})$ from morning until evening is abnormal has been generally accepted as the principal objective criterion for diagnosis. Surprisingly, we can find no evidence in support of this claim apart from measurements of weight variation in studies of idiopathic oedema in small numbers of control subjects, usually under metabolic ward conditions. ${ }^{6-8}$ We therefore measured diurnal and weekly weight and abdominal girth variation in a sample of gynaecological outpatients who also completed a questionnaire on symptoms suggesting fluid retention. This has clarified the relationship between 'normal' weight variation, symptoms of fluid retention and a diagnosis of idiopathic oedema.

\section{Subjects and methods}

Over 8 weeks, new referrals to the gynaecological outpatient clinics of J.A.D. and C.A.F. were asked to complete a two week record of diurnal weight and abdominal girth variation before coming to the 
clinic. Patients were asked to weigh themselves on rising and on retiring in the evening in nightclothes, to pass urine before weighing and to indicate, if appropriate, the days on which they were menstruating. Patients were also requested to record girth measurements at the level of umbilicus after weighing themselves. On attendance, patients completed a questionnaire concerning symptoms suggestive of fluid retention and were asked to record diurnal weight and girth variation for a further two weeks. A drug history was obtained from each patient and a blood sample taken for the estimation of urea and electrolytes, liver function tests, blood glucose and thyroid function. A urine sample was tested for protein and glucose.

Completed charts were examined for maximum daily and weekly weight and abdominal girth variation in each patient. Where possible, maximum daily weight and girth variation within the premenstrual period ( 5 days up to and including the first day of menstruation) was measured. To reduce error, only variations recorded twice or more were included and recordings were made in pounds and inches because of the patients' familiarity with these units of measurement.

The significance of between-group differences in the distributions of diurnal weight and girth variation and between diurnal weight and girth variation and fluid retaining symptoms was assesed by the Chi-squared method.

\section{Results}

Of 142 invitations issued, 43 patients were excluded on grounds of non-attendance, refusal or inability to participate or age. The remaining 99 patients (aged 18-65 years; mean $35 \pm$ s.d. 10 years) completed the symptom questionnaire. Of these, 86 patients were premenopausal and 13 postmenopausal. Their diagnoses (available on request) covered the usual range of non-malignant gynaecological pathology. Clinical and laboratory investigation showed no evidence of major organic disease which might produce abnormal oedema or fluid retention. Eighty-two patients completed satisfactory records of weight and girth variation. Premenstrual weight and girth variation were recorded in 39 patients.

\section{Daily and weekly weight variation (Figure 1)}

Variation in maximum daily and weekly intermenstrual and postmenopausal weight and abdominal girth measurements were similar. These were therefore combined for between-group comparison. Maximum daily intermenstrual and postmenopausal weight variation in each patient appeared normally distributed with skewing to higher values in a small number of patients. Modal weight variation was $2 \mathrm{lb}(0.9 \mathrm{~kg}) ; 6$ patients showed no weight variation. Fifteen patients $(17.8 \%)$ showed maximum daily weight variation of $4 \mathrm{lb}(1.8 \mathrm{~kg})$ or over, fulfilling Thorn's criterion for a diagnosis of idiopathic oedema. Weekly weight variation exceeded daily weight variation but the difference did not reach statistical significance. The distributions of premenstrual and intermenstrual/postmenopausal weight variation did not differ significantly.

\section{Daily and weekly girth variation (Figure 1)}

Most patients showed a daily increase of between one and two inches $(2.5-5.0 \mathrm{~cm})$ in maximum abdominal intermenstrual or postmenopausal girth variation between morning and evening. Only 16 of 82 women showed no variation. Maximum weekly girth variation was significantly greater than daily variation $(P<0.05)$. The distributions of premenstrual and intermenstrual or postmenopausal girth variation did not differ significantly.

\section{Fluid-retaining symptoms (Table I)}

Sixty of 99 patients (61\%) usually experienced swelling at one site and $33(33 \%)$ at two sites. One patient reported intermenstrual swelling at all sites. Complaints of swelling were also reported by the small number of postmenopausal subjects. In the premenopausal group, breast discomfort or swelling was significantly associated with the premenstrual period $(P<0.001)$ and swelling of the fingers and hands with the intermenstrual period $(P<0.001)$.

There was no significant association between complaints of swelling at any site and maximum daily or weekly variation. Four of 15 patients with recorded maximum daily weight variation of $4 \mathrm{lb}$ $(1.8 \mathrm{~kg})$ or more did not complain of swelling at any site. There was a weak association between intermenstrual and postmenopausal daily abdom inal girth variation and subjective complaints of abdominal swelling $(P<0.04)$. There were no significant associations between girth variation and symptoms of swelling at other sites. There was no significant difference in the distributions of weight or girth variation in 22 women taking an oral contraceptive and the remainder of the sample.

\section{Discussion}

A diagnosis of idiopathic oedema depends on a characteristic set of symptoms, the exclusion of major organic causes of oedema and evidence of diurnal weight variation.

Although some patients may show large diurnal weight swings of one stone (6 kg) or more, Thorn's criterion $^{1}$ that weight variation of more than $3 \mathrm{lb}$ 
Daily

(Intermenstrual/ post menopausal)

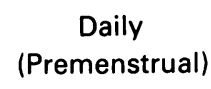

Weekly

(All values)
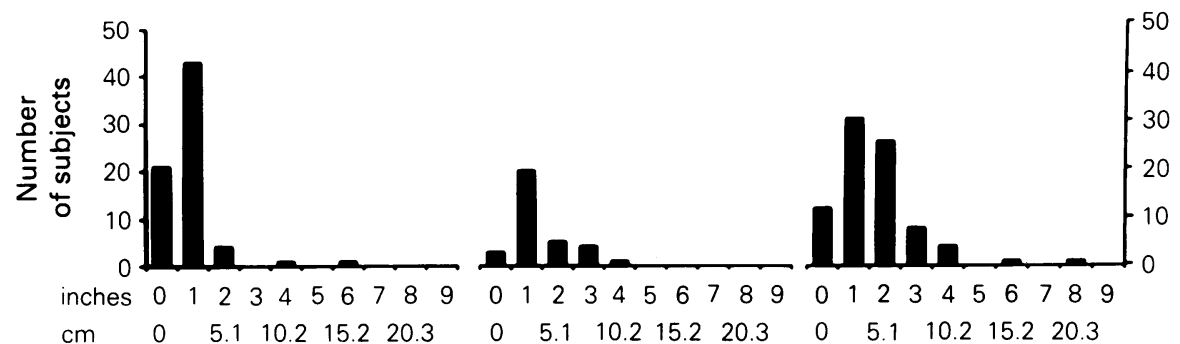

$\begin{array}{llllll}\mathrm{cm} & 0 & 5.1 & 10.2 & 15.2 & 20.3\end{array}$

$\begin{array}{lllll}0 & 5.1 & 10.2 & 15.2 & 20.3\end{array}$

$\begin{array}{lllll}5.1 & 10.2 & 15.2 & 20.3\end{array}$

Maximum morning-evening girth variation

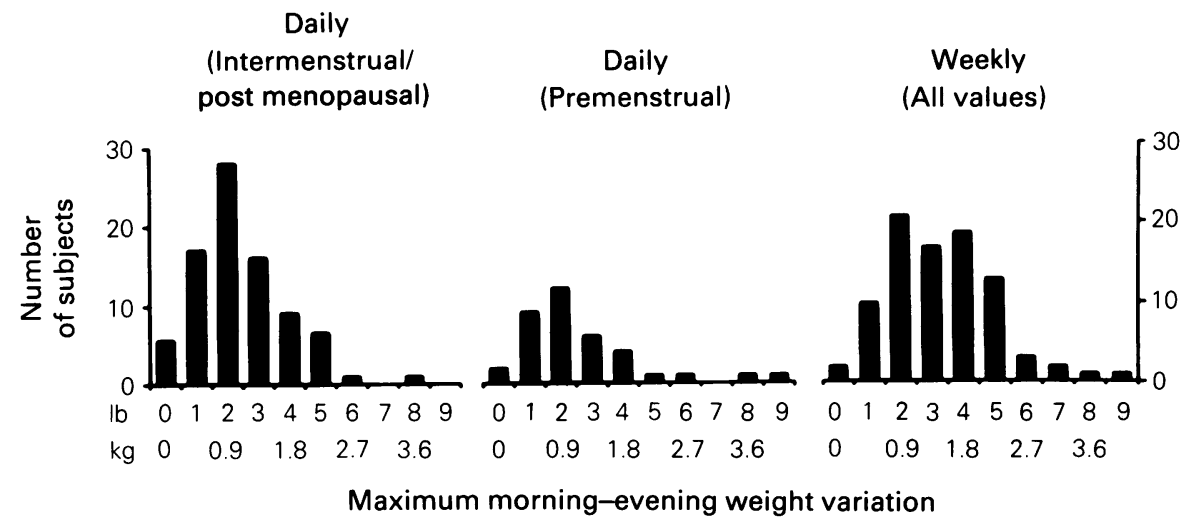

Figure 1 Maximum daily intermenstrual/postmenopausal, premenstrual and weekly morning to evening weight and abdominal girth variation in 82 gynaecological out-patients.

$(1.4 \mathrm{~kg})$ between morning and evening is abnormal has been generally accepted. In studies of the pathophysiology of idiopathic oedema, diurnal weight variation has been previously measured in only small numbers $(7-10)$ of normal subjects, $(6-8)$ usually under metabolic ward conditions. ${ }^{6-8}$ Since diurnal weight variation is increased by physical activity, particularly by the upright posture, and diminished by rest and recumbency, $2,3,6$ weight variation recorded by these subjects is unlikely to be representative of the experience of women under conditions of normal everyday activity.

The present study suggests that symptoms of mild fluid retention are part of most women's everyday experience even though we studied a possibly atypical population (attendees at a gynaecological clinic). Symptoms are experienced with equal frequency premenstrually, intermenstrually and after the menopause. Objective evidence of some daily weight variation was found in $76(93 \%)$ of the 82 women whose self-recorded weight records were examined. Fifteen women
(18\%) recorded weight variations which fulfilled Thorn's criterion for a diagnosis of idiopathic oedema and weekly weight variation of this order was recorded by 34 ( $40 \%$ women in the sample).

The lack of a significant relationship between weight variation and subjective complaints of swelling indicates that factors other than the degree of fluid retention determine perception of this symptom. The majority of women with idiopathic oedema show evidence of psychological disturbance 4 and may have lower thresholds for the perception of somatic sensation than women who are psychologically normal.

Weight variation related to fluid retention appears normally distributed and there is no sharp dividing line between normal and abnormal (Figure 1) once organic causes such as hypothyroidism, drug use, etc., have been excluded. An analogy may be drawn with premenstrual tension in which many women experience mild symptoms as part of everyday experience and a few experience disabling symptoms which lead them to seek medical attention. If Thorn's criterion 
Table I Frequency of symptoms suggesting fluid retention in 99 gynaecological out-patients (86 pre- and 13 postmenopausal)

\begin{tabular}{llrr}
\hline $\begin{array}{l}\text { Usual site of } \\
\text { swelling/discomfort }\end{array}$ & $\begin{array}{l}\text { Usual relationship } \\
\text { to menstruation }\end{array}$ & Number & $\%$ \\
\hline \multirow{3}{*}{ Eyes } & Premenstrual & 14 & 16 \\
& Intermenstrual & 14 & 16 \\
& Postmenopausal & 2 & 15 \\
& Premenstrual & 4 & 5 \\
Face & Intermenstrual & 6 & 7 \\
& Postmenopausal & 1 & 8 \\
& & & \\
Abdomen & Premenstrual & 47 & 55 \\
& Intermenstrual & 38 & 44 \\
& Postmenopausal & 9 & 69 \\
Breasts & Premenstrual & 35 & 41 \\
& Intermenstrual & 7 & 8 \\
& Postmenopausal & 3 & 23 \\
& & & \\
Hands/fingers & Premenstrual & 9 & 10 \\
& Intermenstrual & 24 & 28 \\
& Postmenopausal & 3 & 23 \\
& & & \\
& Premenstrual & 6 & 7 \\
& Intermenstrual & 30 & 35 \\
& Postmenopausal & 3 & 23 \\
\hline & & &
\end{tabular}

for diagnosis is accepted, the present study would suggest that about one in six of the adult female population might be categorized as suffering from idiopathic oedema. If the more conventional criterion of values lying outside the 95 th percentile of the normal distribution is accepted, only two patients showing daily weight variation of more than $5 \mathrm{lb}(2.3 \mathrm{~kg})$ daily would be regarded as abnormal. Hard and fast criteria for abnormal fluid retention are thus difficult to define. A calm and phlegmatic patient may tolerate fluid retention of a severity which might take an anxious and introspective patient to her doctor.

A term such as 'fluid retention' or the 'fluid retention syndrome' seems preferable to the more commonly used term 'idiopathic oedema' which misleadingly implies a discrete disease entity. Fluid retention in women forms a continuous spectrum from normal to abnormal and it seems possible that the aetiological mechanisms producing abnormal fluid retention are an exaggeration of normal mechanisms common to most women. However pathological mechanisms may be operative in the small subset with severe symptoms and extreme weight and girth variation. A risk-factor approach has proved helpful in evaluating the causes of fluid retention in individual patients. Patients with mild fluid-retaining symptoms should not be categorized as suffering from a distinct 'disease' since this may promote hypochondriasis in anxious patients and encourage unnecessary diuretic administration. A stepped-care approach to management should involve initial assessment of the severity of the condition including an evaluation of psychiatric risk-factors. An explanation of the mechanism of normal fluid retention and reassurance that the patient is not suffering from a 'disease' should be provided. Reduction to ideal weight in patients in whom fluid retention is a response to weight gain or obesity and the limited use of diuretics only where this measure fails will control the great majority of patients with idiopathic oedema. ${ }^{3}$

\section{Acknowledgement}

We would like to thank the patients who willingly participated in this study for their co-operation.

\section{References}

1. Thorn, G.W. Approach to the patient with 'idiopathic oedema' or 'periodic swelling'. JAMA 1968, 206, 333-338.

2. Edwards, O.M. \& Bayliss, N.I.S. Idiopathic oedema of women. A clinical and investigative study. $Q J$ Med 1976, 45, $125-144$.

3. Dunnigan, M.G. The recognition and management of the fluid retention (idiopathic or cyclical oedema) and premenstrual syndromes. In: McNaughton, C. (ed) Medical Gynaecology. Blackwell Scientific Publications, Oxford. 1985, pp. 27-54.

4. Pelosi, A.J., Sykes, R.A., Lough, J.R.M. Muir, W.J. \& Dunningan, M.G. A psychiatric study of idiopathic oedema. Lancet 1986, ii: 999-1002.

5. McGregor, G.A., Markandu, N.D., Roulston, J.E., Jones, J.C. \& de Wardener, H.E. Is 'Idiopathic' oedema idiopathic? Lancet 1979, i: $397-400$.

6. Streeten, D.H.P. Idiopathic edema: pathogenesis, clinical features and treatment. Metabolism 1978, 27: 353-383.

7. Kuchel, O., Hamet, P., Cuche, J-L., Tolis, G., Fraysse, J. \& Genest, J. Urinary and plasma cyclic adenosine 3', 5'-monophosphate in patients with idiopathic oedema. J Clin Endocrinol Metab 1975, 41: 181-189.

8. Catania, R.A., Sowers, J.R., Stern, N., Tuck, M.L. \& Paris, J. Altered dopaminergic modulation of sympathetic nervous system activity in idiopathic edema. J Endocrinol Invest 1984, 7: 461-466. 